\title{
OBSERVACIONES DE AVIFAUNA NATIVA EN LA COSTA DE TACNA
}

- Resultados Preliminares -

José Pizarro Neyra'

\section{RESUMEN}

Se presenta información general acerca de algunas aves de la costa de Tacna, basada en reportes de observación de tipo bipotético (sin ninguna captura), hechos entre julio de 1992 y agosto de 1994, en seis lugares ubicados en: orilla marina, bumedales, bosque xerofítico y zona urbana.

La avifauna identificada se clasificó en 10 órdenes y 18 familias. Las especies introducidas no fueron consideradas. Además, están siendo revisadas colecciones privadas $y$ material depositado en colegios de la localidad. Este trabajo ha sido becho para promover la conservación de nuestro patrimonio natural.

\section{INTRODUCCIÓN}

Hasta el año 1990, el Perú era considerado, después de Colombia, como el país con el mayor número de aves descritas en el planeta (más de2410 subespecies que viven en territorio peruano aceptadas hasta 1989), ocupando el primer lugar en el mundo. Según el Centro de Datos para la Conservación (1990), en el Perú se han descubierto 25 especies nuevas para la ciencia entre 1963 y 1983, lo que resulta ser la mayor cantidad de nuevas aves reportadas, comparada con el resto del mundo para el mismo período de tiempo. Además, se han considerado como endémicas del Perú 109 tipos de aves

Sin embargo, hay zonas del país donde los inventarios biológicos están tan atrasados que, probablemente, mucha de la información descrita tendría que ser nuevamente cambiada a la luz de nuevos hallazgos de especies o subespecies de aves, debido al registro de estos vertebrados fuera del área de distribución considerada como la correcta o usual. Una de esas zonas es precisamente el desierto costero del sur del Perú y norte de Chile. Allí, los reportes de aves son escasos y, en general, ningún inventario biológico se ha hecho con seriedad.

\footnotetext{
I Estudiante de ciencias biológicas UNJBG
}

Un aspecto que comúnmente se señala, acerca de los ecosistemas de zonas áridas, es precisamente su baja densidad biótica. Sin embargo, la diversidad biológica no se mide en cantidad por metro cuadrado, sino en consideraciones como: resistencia a cambios climáticos, valor como recurso genético que nos puede dotar, en el futuro, de materia prima para el desarrollo de nuevos procesos científicos y tecnológicos, además de las de orden etnobiológico (las especies silvestres no sólo interaccionan entre ellas, sino también con el medio social, del cual forman parte la economía y la historia del hombre).

Tacna está ubicada en la ecorregión del desierto costero del Pacífico para la que están considerados dos tipos de clima: clima de desierto y clima de estepa. En este trabajo se mencionan las siguientes formaciones vegetales: humedales (con presencia de ciperáceas, gramíneas y plantas acuáticas entre las plantas superiores; y charophitas, cianophitas y bacilariophitas entre la flora criptogámica); bosque xerofítico (con predominancia de leguminosas entre las especies leñosas y descompuestas, amarantáceas, y solanáceas entre las semileñosas y herbáceas); finalmente, la orilla marina (zona supralitoral y mesolitoral) con flora criptogámica característica. Desde el punto de vista zoogeográfico, la zona recibe principalmente la influencia de tres grandes grupos faunísticos provenientes de la provincia desértica, de las vertientes occidentales, y 
del mar frío de la Corriente de Humboldt.

No se han estudiado ecosistemas de lomas. En torno al biotopo urbano correspondiente a los alrededores y la ciudad misma de Tacna, se caracteriza por un perfil urbanístico generalmente bajo, rodeado de áreas verdes escasas, en las que predominan especies forestales como: Piptadenia grata, Eucalyptus globulus, Parkinsonia aculeata y otras como Sphatodea campanulata, Nicotiana glauca y Tecoma fulva.

Debido a las limitaciones de transporte, las observaciones se hicieron con mayor frecuencia en el valle de Tacna. Los otros reportes corresponden a visitas a la zona litoral en cinco puntos diferentes: Llostay, Vila-Vila, Playa Cánepa, Punta Mesa y la zona de humedales de Ite. Este último lugar de observación posee dos áreas bien definidas, una a cada lado del canal que conduce desechos mineros provenientes de Toquepala. El presente trabajo es una parte del proyecto de creación del Grupo Ecológico de la UNJBG, y plantea dos objetivos:

-Promover el estudio y la conservación de las aves de la zona.

-Ampliar el conocimiento existente acerca de los ecosistemas de la zona que son habitados por aves.

\section{DISCUSION DE RESULTADOS}

Se confirma la modificación de límites de distribución de avifauna. Aguilar (1985) no considera a la Costa Sur como área de distribución de $B$. superciliaris. En este trabajo se reporta esta ave para Tacna. Eulidia yarreli, picaflor considerado endémico del norte de Chile, también es reportado para el valle de Tacna en este informe preliminar. Araya y Millie (1986) informan de su presencia en Arica y valles de los alrededores. Meyer de Schauenses (1982) amplía límites de distribución de Thaumastura cora añadiendo "Norte de Chile" al límite definido entre La Libertad hasta Arequipa; sin embargo, hemos encontrado T. cora en el valle de Tacna, ampliando nuestro conocimiento sobre la distribución de dicha ave. El ave observada en mayor abundancia fue Gallinula chloropus, en los humedales de Ite. Esto confirma observaciones y censos realizados por otros investigadores para otras zonas húmedas de la costa: Velásquez (1992) para Cachiche-Ica; de Romaña y Hughes (1985) para Mejía-Arequipa, y Calderón (1988) para el mismo lugar.

Finalmente, se observa que la mayoría de las aves reportadas cumplen un rol dentro del ecosistema humano (urbano o agroecosistema), lo cual nos puede ayudar para preservar este bello recurso faunístico. Veamos:

Insectívoros: C. cinereum, B. ibis, N. cyanoleuca.
Carroñeros: C. aurajota, Larus belcheri...

Oportunistas: Zenaidaasiática, Pelecanusothagus...

Muchas aves, además, propagan semillas por zoocoria o realizan polinización cruzada (p.e. familia Trchilidae).

Se empleó el método de observación directa, correspondiente al llamado tipo hipotético, pues no se realizó ninguna captura.

Los reportes fueron hechos con ayuda de guías de aves según Koepcke (1964), De Romaña y Hughes (1985) y Araya \& Millie (1986). En la identificación final, se siguió a Meyer de Schauensee (1971 y 1982). Además, se revisó la colección ornitológica depositada en el Instituto Pedagógico José Jiménez Borja de Tacna.

Las observaciones incluyeron:

-Características morfológicas externas ( color de plumas, tamaño del pico, color de tarsos, patas y pico,tamaño de tarsos y/o patas. Tipo de pie y longitud total estimada ( de pico a cola).

- Hábitat (orillamarina, hábitatmarino, humedad, terrestre y arborícola).

- Registro auditivo.

2. RESULTADOS En el presente trabajo sólo se exponen resultados de observaciones verificadas, tanto en campo como en la literatura disponible. Las aves han sido agrupadas por Orden y Familia.

\subsection{Orden Passeriformes}

2.1.1. Familia Furnaridae

Cinclodes nigrofumosus taczanowskii (Berlepsch y Stolzman)

Es la única ave del orden Passeres que habita en la orilla marina rocosa. Color general: pardo oscuro con líneas más claras en el pecho y alas.Pico agudo ( $2 \mathrm{~cm}$, aproximadamente). Anisodatilo. Ave observada con longitud total aproximada de $15 \mathrm{~cm}$. Se encontró en la Playa Canepa (orilla rocosa).

\section{Zimmer)}

Phleocryptes melanops brunnescens (

Ave que anida en el humedal izquierdo de Ite. De color pardo, con manchas intensas (ocre-rojo ), y negro; una línea blanca transversal cruza las plumas de la cola. Pico pequeño ( $1 \mathrm{~cm}$ aproximadamente). Longitud total $14-15 \mathrm{~cm}$. Se le observó en la parte izquierda del humedal de Ite.

\subsubsection{Familia Hirundinidae.}

\section{Progne modesta murphyi ( Chapman )}

Golondrina ocasional en playas de Tacna. Color oscuro-azul muy hermoso. Como todas las golondrinas, 
presenta pico muy pequeño y tarsos/pies cortos. Tamaño general grande ( $16 \mathrm{~cm}$ o más ). Se le observó sobre cables de energía eléctrica en el balneario de Llostay.

\section{chapman )}

Pigochelidon cyanoleuca peruviana (

Golondrina de color oscuro, por encima, y blanco sucio por debajo.Tiene hábitos insectívoros. Tamaño general pequeño $(10-12 \mathrm{~cm})$. Se le observó en el cerro Intiorko, Alto de la Alianza y Fundo Los Pichones ( valle de Tacna).

\subsubsection{Familia Tyrannidae}

\section{Pyrocephalus rubinus (Goluld)}

Pequeña ave que se caracteriza por lucir pecho y parte de la cabeza de un color rojo-anaranjado, sólo en el macho. Resto color pardo. Pequeño pico y tarsos, muy delgados. Tamaño general de $12-13 \mathrm{~cm}$. Se le avistó en hábitats arborícolas del valle de Tacna (como chacras y parques) y en la Ciudad Universitaria de la UNJBG.

\subsubsection{Familia Coerebidae:}

\section{Conirostrum cinereum (Lafresnaye y} d'Orbignyi)

Tangara pequeña con partes superiores color gris con tinte oliváceo, mentón y garganta blancas y parte de abajo en colores: gris acanelado o verdoso muy claro. Prefiere buscar su alimento en cornisas de edificios y agujeros de construcciones donde hay arácnidos. Pico agudo y pequeño. Tamaño general pequeño $12-13 \mathrm{~cm}$. Observado en viviendas antiguas de madera o adobe ( Villa Violeta y Quinta Casanova) o edificios con agujeros (local central UNJBG) ubicados en el valle de Tacna.

\subsubsection{Familia Fringilidae:}

Zonotrichia capensis peruviensis (Lesson)

Ave muy conocida en el folclor de Tacna. No tiene cresta como algunos afirman. El macho posee en la cabeza plumas de la corona eréctiles que sobresalen por ser de color gris. Posee un " antifaz " que lo conforman dos líneas laterales que cruzan la cabeza y son de color negro. El pecho es blanquecino. Las alas tienen coloración blanca, canela y negra. Tamaño general de $12-14 \mathrm{~cm}$. Observado en todo el valle de Tacna.

\section{Spinus magellanicus paulus (todd)}

Pequeña ave reconocida por su plumaje amarillo-negro y su trino de características melodioso y continuado. En esta especie, sólo la cabeza está teñida de negro completo; el resto del cuerpo presenta color amarillo u oliváceo conmanchas oscuras y amarillas, casi sin interrupción en el vientre. Longitud total 12-
$13 \mathrm{~cm}$. Encontrado en chacras y zonas arboladas de la ciudad de Tacna.

\subsection{Orden Pelecaniformes}

2.2.1 Familia Pelelcanidae:

Pelecanus Occidentalis thagus (Molina).

Pelícano endémico del mar frío de la Corriente de Humboldt. Mitad anterior de la cabeza y garganta de color blancos. Cuello gris. Dorso gris-plateado. Mitad inferior del cuerpo, pardusco, con manchas blancas y grises. Coloración de cabeza y cuello puede cambiar a blanquecino en época de cría. Tarsos cortos y patas con membrana interdigital. Pico gran de ( $25 \mathrm{~cm}$ aprox.), con membrana extensiva. Longitu d total d e 1,40-1,50 $\mathrm{m}$. Avistado en todo el litoral, pero común en caleta VilaVila.

\subsection{Orden Micropodiformes.}

2.3.1 Familia Trochilidae:

Thamastura cora ( Lesson y Garnot).

Considerado hasta 1982 como endémico del Perú. Picaflor pequeño que se le identifica por su larga cola (plumas rectrices de hasta $8 \mathrm{~cm}$ de largo), presente en el macho de esta especie.Plumaje verde en el dorso, pecho oliváceo y mancha violeta en la garganta. Pico corto,tamaño general $11-12 \mathrm{~cm}$. Observado en zona urbana y chacras aledañas de Tacna.

\section{Myrtis fanny ( Lesson )}

Picaflor pequeño similar al anterior en cuanto a su coloración. Dorso con tonalidaes atornasoladas. Pico másgrande ( $1,5 \mathrm{~cm}$ aprox.); coloración nupcial en el macho con tono azulado en el cuello y una franja violeta en la garganta. Tamaño general $7,5 \mathrm{~cm}$. Observado en zona urbana y bosque xerofítico nativo.

\section{Eulidia Yarrelii ( Bourcier)}

Picaflor endémico del norte de Chiley observado también en Tacna. Muy parecido a M. Fanny. Se diferencia por tener pico más pequeño ( poco más de $1 \mathrm{~cm}$ ). Además, en el macho las plumas rectrices centrales de la cola son muy cortas, comparadas con las de $\mathrm{M}$. Fanny. El macho también presenta garganta violeta. Longitud total casi $8 \mathrm{~cm}$. Avistado en zona urbana y bosque xerofítico nativo de Tacna.

\section{Rodophis vesper vesper (lesson)}

Picaflor mediano con cola similar a la del anterior (rectrices centrales más cortas que las externas). Dorso verde atornasolado. En el macho también se presenta una garganta violeta algo más encendida. Plumaje ventral blanquecino. Rabadilla ocrácea y rectrices oscuras o pardas. Pico largo (un poco más de $3 \mathrm{~cm}$ ) y curvado hacia abajo. Longitud total $10 \mathrm{~cm}$ aproximadamente. Registrado para el valle de Tacna en zona urbana (parques y jardines) y bosque xerofítico nativo. 


\subsection{Orden Falconiformes}

Familia Falconidae:

\section{Falco sparverius peruvianus (Cory)}

Halconcito bastante común en bosquexerofítico y zona urbana de Tacna. Plumaje de la cabeza con "gorro" negro y manchas blancas y negras. Franja transversal a las rectrices de la cola color oscuro. Pecho y cubiertas ventrales de las alas de color más claro. Dorso del cuerpo ocre con manchasnegras. El macho luce alas con tonos azulados. Las alas son en forma de "boomerang". Pico pequeño y ganchudo. Longitud total aproximado de $25 \mathrm{~cm}$. Observado en paisaje urbano (nidos sobre antenas de radio o postes altos) y bosque xerofítico (cerca de Magollo, Feria Agronómica y fundo Los Pichones).

\section{Familia Cathartidae:}

\section{Cathartes sura jota (Molina)}

Buitre bastante conocido, en especial en la zona litoral de Tacna. Partes desnudas de la cabeza de color rojo. Resto del cuerpo con plumaje negro, excepto plumas remiges de tonos mucho más claros en las alas. Pico robusto y pequeño. Longitud total $70 \mathrm{~cm}$ aprox. Avistado cerca de concentraciones urbanas del litoral (Llostay, Vila-vila) y sobre promontorios rocosos y carteles publicitarios.

\subsection{Orden Charadriiformes}

2.5.1 Familia Charadriidae: (Cabanis)

\section{Charadrius alexandrinus occidentales}

Pequeño chorlo frecuentemente encontrado en playas arenosas del litoral de Tacna. Plumaje del dorso color gris-arena. Pequeñas manchas de color negro a cada lado del pecho. Igual coloración en zona auricular y frontal de la cabeza. "Collar" blanco interrumpido a la altura del pecho. Pecho y parte ventral color blanco. Pico y patas color oscuro. Tamaño general $14-15 \mathrm{~cm}$. Avistado en playas arenosas desde Llostay hasta Ite.

\section{Charadrius vociferus peruvianus (Chapman)}

Chorlo mediano caracterizado por su trino escandaloso. Presenta una franja que cruza la cabeza, pasando por el ojoque es de color oscuro. "Collar" blanco completo. Parte del pecho color negro, con un segundo collar blanco. Vientre blanco. Dorso gris-arena con tonos ocres, negros y blanquecinos en la cola. Patas de color naranja intenso. Pico mediano color oscuro. Longitud total $24-25 \mathrm{~cm}$. Observado en playas arenosas de Llostay.

\subsubsection{Familia Laridae:}

\section{Larus belcheri (Vigors)}

Gaviota mediana muy común. Alas negras con plumas remiges blancas. Cola con rectrices mayoritariamente negras. Resto del cuerpo con plumaje blanco. Colores oscuros tenues en cabeza y cuello en invierno. Pico con manchas negras y rojas en la punta y el resto color amarillo. Patas amarillas. Longitud total $45-50 \mathrm{~cm}$. Presente en todas las estaciones de observación en el litoral.

Larus dominicanus (Lichtenstein)

Gaviota grande. Similar a L. Belcheri, pero sin rectrices negras en la cola. Pico amarillo con mancha naranja en la punta. Patas amarillas. Longitud total entre 50 y $55 \mathrm{~cm}$. Observada en Punta Mesa y LLostay.

Larus modestus (Tschudi)

Gaviota pequeña de plumaje plomo grisáceo en todo el cuerpo. Algunas plumas remiges de las alas color negro y blanco. Cabeza de tono blanquecino. Pico y patas oscuras. Longitud total $40 \mathrm{~cm}$ aprox. Avistada en playas cercanas de todo el litoral.

\section{Larus pipixcan (Wagler)}

Gaviota pequeña. "Gorro" negro en la cabeza. Al volar sele distinguen alas grises con plumas remiges negras en sus extremos. Resto del plumaje color blanco. Pico y patas oscuras. Longitud total $30-35 \mathrm{~cm}$. Avistada en todo el litoral, a excepción de Vila-Vila.

\subsubsection{Familia Haematopodidae:}

Haematopus ater (Vieillot y Oudart)

Ave mediana con plumaje general color negro. Patas y pico color naranja intenso. Pico largo y tubular (7cm aprox.) y tamaño general entre $45-50 \mathrm{~cm}$. Observado en grupos al sur de Llostay, en playas arenosas. Solitario en orilla rocosa.

\subsubsection{Familia Burhinidae:}

\section{Burhinus superciliaris (Tschudi)}

Chorlo cabezón de hábitos terrestres. Plumaje del abdomen y parte inferior del pecho color blanquecino. El resto del cuerpo de coloración pardo-terroso con manchas grises. Algunas plumas remiges primarias de las alas y rectrices de la cola de color oscuro. Garganta también blanca. Típica franja blanca que parte de la base del pico y se aproxima a la nuca. Tarsos largos y pies pequeños. Pico con mancha negra en la punta. Longitud total $40 \mathrm{~cm}$ aprox. Avistada en cerro Intiorko, CPM La Natividad y fundo Los Pichones (valle de Tacna).

\subsection{Orden Columbiformes}

2.6.1. Familia Columbidae:

\section{Zensaida asistica meloda (Tschudi)}

Paloma abundante en parajes arbolados del valle de Tacna. Coloración general gris-plomiza, línea blanca en cada ala enforma de media luna. Parte desnuda alrededor de los ojos color azulado. Patas y tarsos medianos color anaranjado intenso. Pico color oscuro. Tamaño general $25 \mathrm{~cm}$ aprox. Avistada en zona urbana (techos de las casas y parques) y en chacras del 
valle de Tacna.

Eupelia cruziana (Knip y Prevost)

Pequeña paloma de coloración pardo-grisácea.

Algunas plumas de la cola color negro. Pequeñas manchas negras en cubierta dorsal del ala. Algunas plumas negras en cubierta ventral del ala. Pico amarillo con punta oscura. Patas anaranjadas. Ojos rojos. Longitud total $16-18 \mathrm{~cm}$. Observada tanto en bosque xerofítico nativo, como en zonas arboladas dentro del perímetro urbano de Tacna.

\subsection{Orden Anseriformes}

2.7.1. Familia Anatidae:

\section{Anas bahamensis (Linnaeus)}

Pato considerado residente de zonas húmedas de la Costa. Presenta una gran mancha blanca que ocupa la mitad de la cara y la garganta, en forma de semicírculo. El resto del plumaje es pardusco con manchas negras y blancas. Franja negra transversal a remiges dorsales del ala. Patas color café. Pico oscuro con manchas amarillas/naranja en la base. Longitud total $40 \mathrm{~cm}$ aprox. Observado en los humedales de lte.

\subsection{Orden Gruifomes}

2.8.1 Familia Rallidae:

Gallinula chloropus pauxillia (Bangs)

Gallareta abundante en los humedales de Ite. Plumaje general de color plomo oscuro. Plumas de los flancos y de la rabadilla de color blanco. Patas de dedos largos y de colorverde. Pico presenta placa córnea en su nacimiento y es de color amarillo. Longitud total 35 $40 \mathrm{~cm}$ aprox. Observada en el lado izquierdo de los humedales de lte (donde existen lagunillas con formaciones vegetales en las que predominan ciperáceas) y en los gramadales del lado derecho (donde predomina la verdolaguilla, S. Fruticosa).

\subsection{Orden Ciconiiformes}

\subsubsection{Familia Ardeidae:}

Bubulcus ibis ibis (Linnaeus)

Garza pequeña que se le encuentra asociada a la presencia de ganado. Plumaje entero color blanco. Pico amarillo. Patas color naranja. Plumaje de apareamiento, muestra plumas de cuello y pecho de tono rosado-intenso. Apariencia "ovalada" desde lejos Longitud total $40-45 \mathrm{~cm}$. Frecuenta gramadales del lado derecho en Ite.

\subsection{Orden Estriglformes}

\subsubsection{Familia Strigidae:}

Speotyto cunicularia nanodes (Berlepsh y Stolzman)

Lechuza de hábitos terrestres. Plumaje de color terroso-pardo con manchas blancas más frecuentes en parte ventral y alrededor de los ojos. Longitud total $20-25 \mathrm{~cm}$. Habita espacios abiertos con presencia de arbustos xerofíticos y herbáceas (valle de Tacna).

\section{BIBLIOGRAFIA}

AGUILAR, Pedro. 1985, Fauna de las lomas costeras del Perú.Boletín de Lima, VII (41).

ARAYA B. y G. MILLIE. 1986, Guía de Campo de las Aves de Chile.Ed. Universitaria, SantiagoChile.

CDC-PERU. 1990, "Diversidad biológica del Perú". Boletín Amigo Guarda. Año V(2-3): 1. LimaPerú.

CALDERON A. 1988, Censo de poblaciones delS.N. de MejíaEI Volante Migratorio 7(13): 19-21.
DE ROMAñA M. y R. HUGHES. 1985. Lista de Aves observadas en el S.N. de Mejía. PRODENA, Arequipa-Perú.

KOEPCKE M. 1964, Las Aves del Departamento de Lima.

MEYER DE SCHAUENSEE R. 1982, A Guide to the birds of Southamerica. Reprinted by ICBP,

$$
\text { E.E.U.U. }
$$

VELASQUEZ V. S/F Aves de las lagunas de oxidación de Cachiche-Ica.EI Volante Migratorio 10(19). 
Buliaia yarrelii

Picaflur de Arica
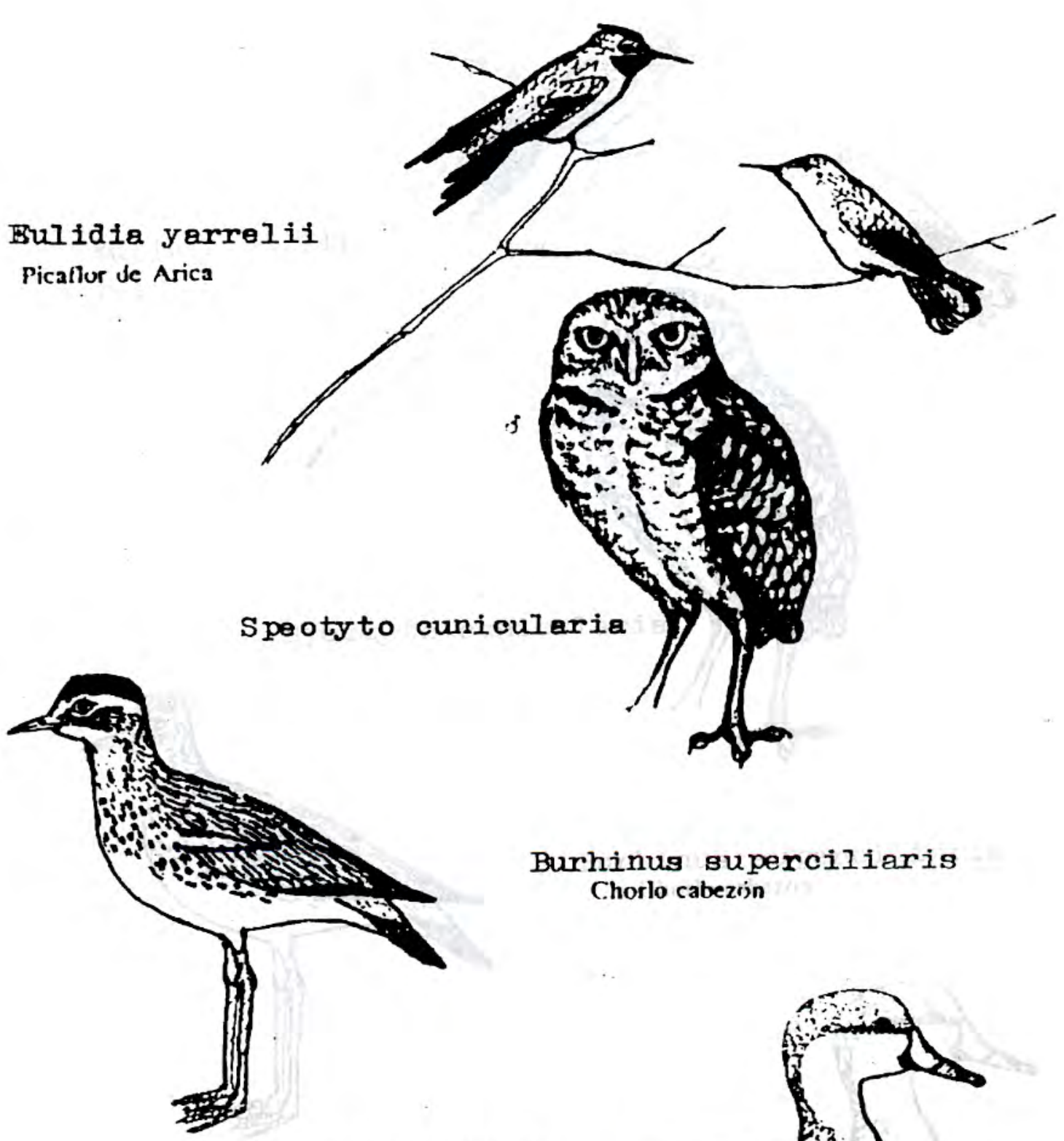

Burhinus superciliaris Chorlo cabezion

Anas bahamensis 